\title{
Stable Data Transmission Method Based on Cluster Partition Sensing Head in Wireless Sensor Network
}

\author{
Limou $\mathrm{Xu}^{1}$, Changyun $\mathrm{Li}^{2}$ and Junfeng $\mathrm{Man}^{2}$ \\ ${ }^{1}$ Department of Information Engineering, Zhongshan Torch Polytechnic, \\ Zhongshan 528437, China \\ ${ }^{2}$ School of Computer and Communication, Hunan University of Technology, \\ Zhuzhou 412007, China \\ E-mail: $14309241 @ q q . c o m$
}

\begin{abstract}
Through analyzing and researching the cross-layer model proposed in traditional literatures for the data transmission in wireless sensing networks and the improved version thereof, the problem of the inaccurate data slot selection is found in current cross-layer data transmission mechanism. Then, mini-slot is adopted to improve data frame structure and reduce data transmission conflict ratio, thus to reduce network throughput and energy consumption at the same time. Moreover, the slot function selection process and the mathematical derivation process are provided for the multi-hop network. However, due to the limited experimental conditions at present, the improvement for test site selection, experiment size, etc. can be achieved in future.
\end{abstract}

Keywords: Wireless sensor network; Energy consumption; routing protocol; Data monitor window

\section{Introduction}

Wireless sensor networks (WSNs) have been widely applied in various fields such as military affairs, medical treatment and transportation and played an irreplaceable role [1 2]. Currently, most sensors are powered by batteries and these sensors are required to work continuously for several months and even for several years in most application fields. Since the sensors are deployed in special areas, it is very difficult and expensive to replace these sensors. In other words, the volume of the data transmitted by the sensors is limited. Therefore, how to adopt the limited battery capacity to give play to the sensor function is a significant research [3]. For the research on the service life prolonging of WSNs, besides the data transmission energy consumption limitation, the common method is to convert the sensor nodes not used for data transmission into the sleep state so as to significantly reduce energy consumption under the precondition of not influencing data transmission. Open system interconnection (OSI) model is usually used to designate the protocol system structure, but it is difficult to use the traditional layer model without energy consumption memory in WSNs. In order to solve such problem, some scholars have proposed the cross-layer model to improve the overall performance of WSN system [4]. The cross-layer model has been improved and researched in previous algorithms from different angles and certain effects have also been obtained, but the data slot selection has not yet been accurately considered. In order to further improve the slot selection accuracy and rationality of the cross-layer model, the slot data frame is improved in this paper on the basis of mini-slot to design the energysaving WSNs data transmission mechanism based on mini-slot cross MAC routing protocol slot superposition. 


\section{CLRP-MS Data Window}

\subsection{Basic Principle for Slot Selection}

$N$ is assumed as the sensor quantity, $L_{i}$ is assumed as the straight path loss between sensor $i$ and signal collector, and $L_{\max }$ is assumed as the maximum path loss of the design system. Additionally, $y=f(x)$ is a decreasing function with the value and result thereof in the interval of $[0,1]$, and function $f$ represents the data transmission and access characteristics of the sensor and the main function of the process.

Sensor $i$ is assumed to have one data packet to be transmitted, wherein such data can be generated by sensor $i$ or by another sensor $j$ but shall be forwarded by sensor $i$. The calculation formula for calculating the slot of sensor $i$ [9] is as follows:

$$
s_{i}=\left[s f\left(L_{i} / L_{\max }\right)\right]
$$

Sensor $i$ takes $s_{i}$ as the reference to transmit slot. Due to the decreasing property of function $f$, when sensor $i$ is far away from the signal collector (beacon), slot $s_{i}$ is located at the starting position of the data transmission window; or else, it is located at the end of the window, as shown in Figure 1. Subsequently, the data are transmitted from the farthest node to the nearest node, and such transmission mode is favorable for improving the data forwarding rule and reducing the transmission energy consumption.

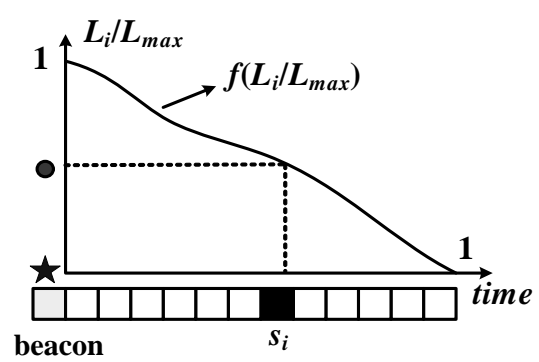

Figure 1. Data Transmission Window

According to the above definition, the value range of slot $s_{i}$ is $[0, S-1]$. If two or more nodes generate or transmit data packets at the same slot $s_{i}$, then relevant conflict will be generated. In such case, the node needs to forward the data packet again at the next data frame, but if the next data frame is adopted for data forwarding at the same slot, then new conflict will be generated. Like this, repeated conflicts can not only waste the network throughput, but also increase the energy consumption. In order to reduce data conflict risk, a random slot selection mechanism is proposed in this paper and the improved version thereof is also provided.

\subsection{CLRP-MS Data Transmission Window}

The random selection process can increase the length of the transmission window in order to effectively reduce the probability of multi-data transmission at the same slot. $l$ is assumed as the discrete random integer variable and $g$ is assumed as the probability density function thereof. Then, the data transmission slot of sensor $i$ is as follows: 


$$
t_{i}=\max \left(0, \min \left(s_{i}+l_{i}, S-1\right)\right)
$$

Meanwhile, $l_{\min }$ and $l_{\max }$ are respectively assumed as the minimum value and the maximum value of random variable $r$. If $l_{\min } \leq 0$ is true, then all possible data transmission slots are located in the interval of $\left[s_{i}+l_{\min }, s_{i}+l_{\max }\right]$, as shown in Figure 3.

In practical operation, $t_{i}$ value range is the whole interval of $\left[s_{i}+l_{\min }, s_{i}+l_{\max }\right]$, where $t_{i} \in\left[s_{i}+l_{\text {min }}, s_{i}+l_{\text {max }}\right]$. But for the above practice, the random signal selection has large fluctuation which is unfavorable for the stability of the data transmission process. Therefore, the above random selection mode shall be improved as follows: the slot is detailed, and each slot is composed of a series of min-slots (as shown in Figure 2 (b)), and each min-slot has a certain duration which is related to network communication delay, so the original slot duration is the sum of the data transmission time and mini-slot durations. Before data transmission, sensor node $i$ calculates $t_{i}=s_{i}$ and then selects the mini-slots; the data will be transmitted only when sensor node $i$ senses the idle channel at the starting position of the minislot. On this basis, the data transmission conflict probability is reduced along with the increase of the mini-slot quantity.

Mini-slot quantity $M$ is assumed to follow the uniform distribution, and $N$ is assumed as the quantity of the nodes accessing the same slot. If the sensor nodes are assumed to have the capability for mutual communication, without any conflict among the first mini-slots of these nodes, then the data transmission slot conflict probability can be calculated as follows:

$$
P_{c}=1-\frac{N \sum_{i=1}^{N}((M-i) / M)^{N-1}}{M}
$$

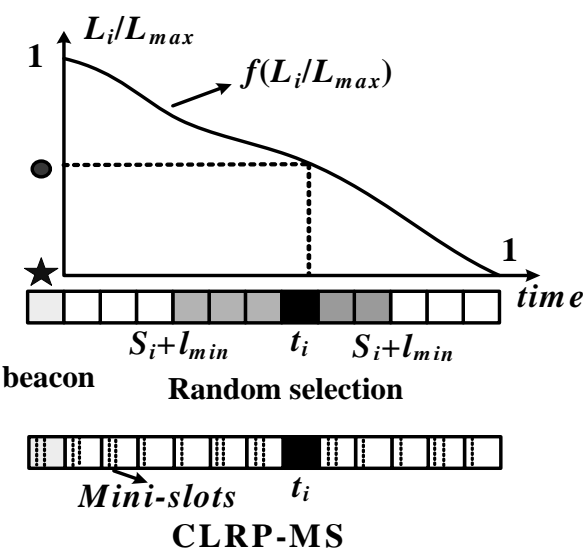

Figure 2. Random Selection and CLRP-MS Slot Superposition

Each node may forward the data generated by the node farther away from the beacon, so it is necessary to continuously monitor this transmission channel within a certain period. Meanwhile, the monitoring time must be long enough for effectively monitoring the relay process, but must be also minimized for reducing energy consumption.

For node $i$ searching the forwarding data, due to the succession of the transmission process, there is no need to monitor the slot data after $s_{i}+r_{i}$; since the slots with the number less than that of $s_{i}$ are located far away from node $i$, the data 
monitor window shall be slightly smaller than or approximate to $s_{i}$. After parameters $\delta$ and $W$ are defined, the monitoring internal shall be $\left[s_{i}-\delta-W, s_{i}-\delta\right.$ ], as shown in Figure 3. The sensors in the monitor window are under the active state, and other sensors are under the sleep state.

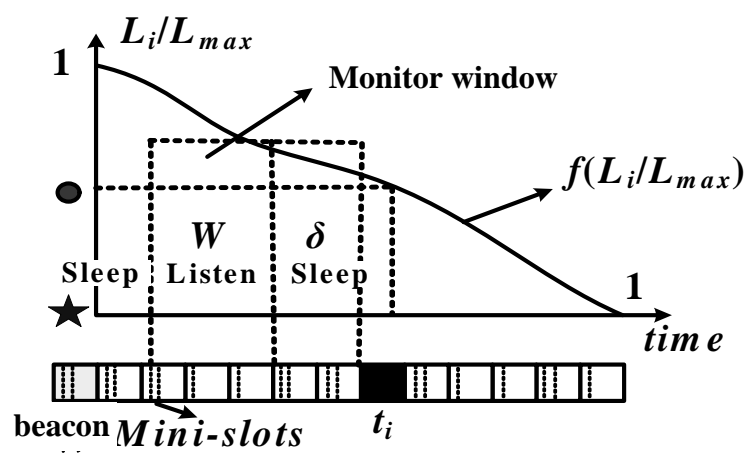

Figure 3. Monitor window

\section{Selection of Slot Function $\mathbf{f}$}

In order to better optimize the data transmission and access mechanism, function $f$ must be carefully selected. The first selected target is the data transmission volume of all slots $s_{i}$ in the equilibrium data transmission window; if nodes are uniformly distributed in the area with the radius as $R$, then the cumulative distribution function of the non-shadow area with the radius as $r$ is $1-(r / R)^{2}$. Then, the cumulative distribution function of the path loss is $1-\left(L_{i} / L_{\max }\right)^{2 / \alpha}$, where $L_{\max }=R^{\alpha} / k$. Specifically, in 1-hop system without data forwarding, function $f$ can be selected as follows:

$$
f\left(L_{i} / L_{\max }\right)=1-\left(L_{i} / L_{\max }\right)^{2 / \alpha}
$$

The above selection mode is mainly adopted to ensure that all slots $s_{i}$ have the equal probability for being selected. Therefore, the following Theorem 1 can be obtained.

Theorem 1: In a data forwarding system, function ${ }^{f}$ can be selected as follows:

$$
f\left(L_{i} / L_{\max }\right)=1-\left(L_{i} / L_{\max }\right)^{1 / \alpha}
$$

Proof: For simplification and generality, the network radius is assumed as 1 , as shown in Figure 4. $\rho(u)$ is assumed as the sensor density $(0<u \leq 1)$ within distance $u$, and then the cumulative probability density thereof can be calculated as $2 \rho(u) u d u$. Meanwhile, if $\tau(r)$ is assumed as the cumulative distribution function of the sensor $r$ away from the beacon, then the following formula can be obtained.

$$
\tau(r)=\frac{\int_{0}^{r} 2 \rho(u) u d u}{\int_{0}^{1} 2 \rho(u) u d u}
$$

If $\bar{f}(x)=1-f(x)$ is set and the path loss is set as $h(r)=L_{\max } r^{\alpha}$, then the cumulative probability density function of slot $s$ is as follows:

$$
F_{s}=\tau\left(h^{-1}\left(L_{\max } \bar{f}^{-1}(s)\right)\right)
$$


Due to the uniform distribution, $f$ can be found to meet $F_{s}(s)=s$ and the following formula can be obtained:

$$
\bar{f}(x)=\tau\left(h^{-1}\left(L_{\max } x\right)\right)
$$

Hops model is simplified as shown in Figure 2. The average transmission distance of the nodes is $\beta<1$ and area $A_{i}$ of the annular part is calculated as follows:

$$
A_{i}=\pi \beta(2 \alpha-\beta+2 \beta i)
$$

Then, the total data arrival rate for annular part $i$ is as follows:

$$
\chi_{i}=\lambda \rho \pi\left(1-(\alpha+\beta(i-1))^{2}\right)
$$

Where $\lambda$ is the new data packet arrival rate of the node. Then, the total density of the real and virtual sensors is as follows:

$$
\rho_{i}=\frac{\chi_{i}}{A_{i}}=\frac{\lambda \rho\left(1-(\alpha+\beta(i-1))^{2}\right)}{\beta(2 \alpha-\beta+2 \beta i)}
$$

For $r=\alpha+\beta(i-1 / 2), i=(2 r-(2 \alpha-\beta)) / 2 \beta$ is true and can be put into the above formula to obtain the following formula:

$$
\rho(r)=\frac{\left(\begin{array}{l}
1-(\alpha-\beta)^{2}-0.25(2 r-(2 \alpha-\beta))^{2} \\
-(\alpha-\beta)(2 r-(2 \alpha-\beta))
\end{array}\right)}{\beta(2 \alpha-\beta)+\beta(2 r-(2 \alpha-\beta))}
$$

If $r \geq \alpha-\beta / 2$ is true, then the following formula can be obtained:

$$
\rho(r)=\frac{1}{2}-\frac{r}{2 \beta}+\frac{\left(4-\beta^{2}\right)}{8 \beta r} \approx \frac{1}{2 \beta r}
$$

According to Formulae (13) and (6), $\tau(r)=r$ is true; according to Formula (8), $\bar{f}(x)=x^{1 / \alpha}$ is true. In conclusion, the following formula can be obtained.

$$
f\left(L_{i} / L_{\max }\right)=1-\left(L_{i} / L_{\max }\right)^{1 / \alpha}
$$

Q.e.d

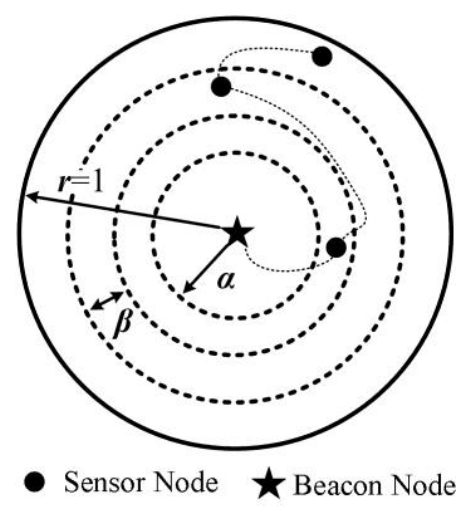

Figure 4. Simplified Data Transmission Model

\section{Simulation Experiment and Analysis}

\subsection{Simulation Experiment Design}

A simplified single-hop slot-aloha access process is considered in this paper, and the spread spectrum technology is adopted to improve the transmission range of the 
sensor in order to make it able to arrive at the beacon node. $S F$ is set as the spread spectrum factor. Compared with the non-spread spectrum network, the data transmission time shall be multiplied with $S F$, and in order to keep the same data frame period, the slot quantity of the data frame shall be correspondingly multiplied with $S F$. Such wireless parameters [10] as transmission power, noise coefficient and data rate are set according to IEEE802.15.4, and the power of the beacon node is $100 \mathrm{~mW}$.

Single-hop transmission needs $20 \mathrm{~dB}$ gain; the spread spectrum factor is usually nth power of 2, so this factor is selected as $S F=64$. The path loss is assumed as $r^{\alpha} / k$, where $\alpha=3$ and $k=3.16210^{-10}$. The transmission distance of the sensor node is $2 \mathrm{~m}$, the transmission distance of the beacon node is $4 \mathrm{~m}$, the experiment radius is $5 \mathrm{~m}$ and there are 32 sensors, as shown in Figure 5.

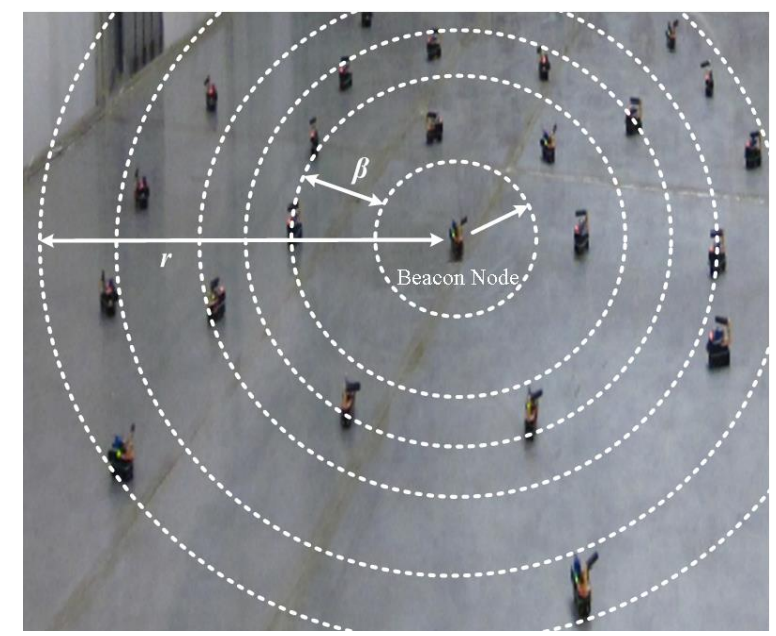

Figure 5. Experiment site layout

The sensor under the sleep state is assumed to not consume any energy, the power dissipation of the sensor under the idle state is $10 \mathrm{~mW}$ and the power dissipation of the sensor for data transmission is $60 \mathrm{~mW}$. The duration of the data frame is $82.16 \mathrm{~ms}$, and when the data frame starts to transmit a data packet with the size of 160bit, the duration change range of each slot is $0.66 \sim 1.3 \mathrm{~ms}$. There are 8 mini-slots, and the duration of each mini-slot is $2 \mu \mathrm{s}$, and the whole experiment lasts for 1000s. The hardware equipment is as follows: CPU i3 $2.4 \mathrm{GHz}$, RAM $6 \mathrm{G}$, OS Win7. The simulation software is matlab2012a.

\subsection{Algorithm Simulation Comparison}

The common analysis indexes [11] include bandwidth availability ratio under different loads, transmission delay, energy consumption, etc., wherein the load refers to the quantity of the data packets at each slot and is expressed by function $\lambda, N$ is the quantity of the sensor nodes in the system, $T_{\text {frame }}$ is the duration of the data frame, so the offered load is $N \lambda T_{\text {frame }} / S$. Due to the existence of the spread spectrum factor, the offered load of the proposed algorithm is $64 N \lambda T_{\text {frame }} / S$. PLOSA algorithm and aloha data frame algorithm [12] are selected as the comparison algorithms. Specifically, the simulation comparison results of the three indexes ---- packet loss probability, average node energy consumption and transmission delay are as shown in Figures 5 (a) (c). 


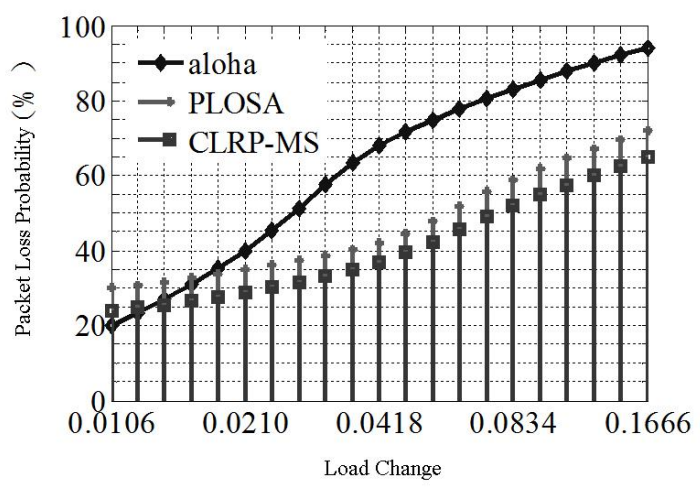

(a) Packet loss probability

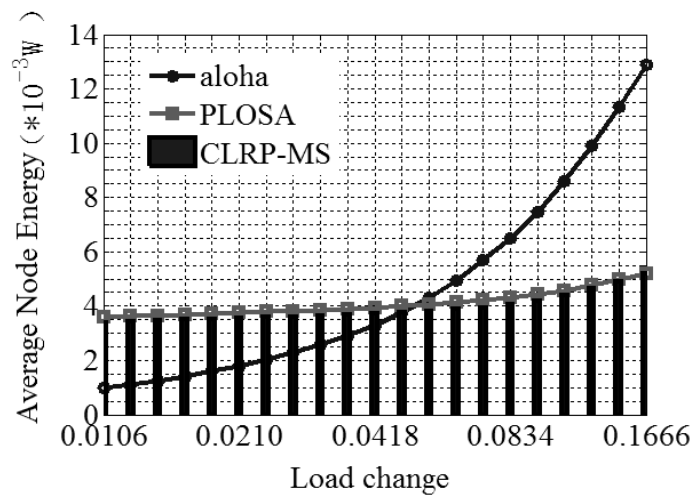

(b) Average node energy consumption

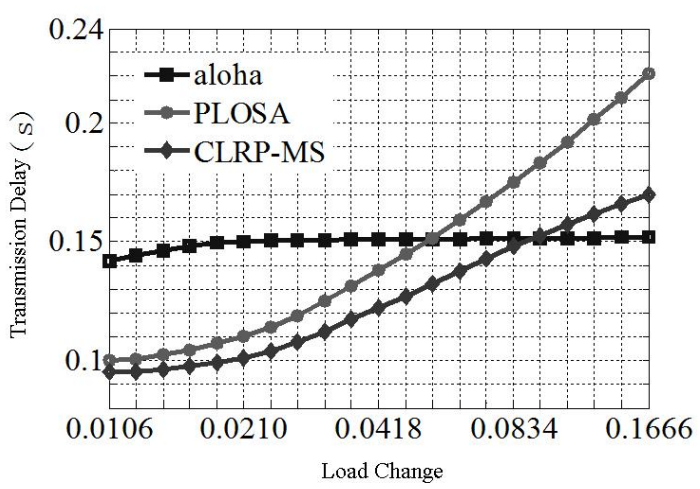

(c) Transmission Delay

Figure 6. Changes of the Three Indexes along with the Load

The simulation curves of the three algorithms for the three indexes ---- packet loss probability, average node energy consumption and transmission delay are as shown in Figure 6 (a) (c). According to Figure (a), the packet loss probability of CLRP-MS algorithm is slightly different from that of PLOSA algorithm but is overall obviously better than that of PLOSA algorithm, and such advantage is slightly expanded along with the load increase. For example, when the load is selected as 0.0106/sensor, the packet loss probabilities of PLOSA and CLRP-MS algorithms are respectively $29.54 \%$ and $24.37 \%$, with the difference as $5.17 \%$; but when the load is increased to 0.1666 /sensor, the packet loss probabilities of PLOSA and CLRP-MS algorithms are respectively $72.19 \%$ and $65.81 \%$, with the difference as $6.38 \%$. Under low load, the packet loss probability of aloha algorithm is slightly better than those of PLOSA and CLRP-MS algorithms, but along with the load increase, the packet loss probability is rapidly increased, thus indicating that aloha 
algorithm is highly sensitive to the load. According to Figure (b), the average node energy consumption of CLRP-MS algorithm is significantly approximate to that of PLOSA algorithm, and along with the load increase, the power dissipations of the two algorithms tend to be the same; but the average node energy consumption of aloha algorithm is also sensitive to the load and presents the exponential increase tendency along with the load increase. According to Figure (c), the transmission delay of the three algorithms is increased along with the load increase. Under low load, CLRP-MS algorithm has the minimum delay, but under high load, aloha algorithm has gradually saturated transmission delay and is superior to the proposed CLRP-MS algorithm mainly for the following reason: for 1-hop system, the sensor nodes at any slot in the network have the same data transmission time which is equal to the data frame transmission time, but for multi-hop process, the data transmission time is related to the sensor node slot.

\section{Conclusion}

In allusion to the large energy consumption of the traditional data transmission mechanism, which is unfavorable for prolonging the service life of WSNs (Wireless Sensor Network), the energy-saving WSNs data transmission mechanism based on mini-slot cross MAC routing protocol slot superposition is proposed in this paper. Firstly, in allusion to the large data conflict probability, network throughput waste and energy consumption increase in the fixed data transmission slot selection mechanism, a random slot selection mode is proposed and meanwhile the improved version based on mini-slot and the data monitor window selection mode are also provided in order to improve data transmission stability and reduce energy consumption. Secondly, mathematical analysis is adopted to give the optimal slot function selection mode in order to improve slot selection accuracy and rationality. Finally, the comparison experiment carried out for the experiment object shows: compared with PLOSA algorithm and aloha algorithm, CLRP-MS algorithm has better data transmission performance and lower energy consumption.

\section{Acknowledgement}

The research is supported by Project supported by National Science and Technology Ministry, China (Grant No.2013BAJ10B14-5).

\section{References}

[1] J. Hu and Z. Gao, "Modules identification in gene positive networks of hepatocellular carcinoma using Pearson agglomerative method and Pearson cohesion coupling modularity", Journal of Applied Mathematics, 2012 (2012).

[2] D. Jiang, Z. Xu and Z. Chen, "Joint time-frequency sparse estimation of large-scale network traffic", Computer Networks, vol. 55, no. 15, (2011), pp. 3533-3547.

[3] J. Hu, Z. Gao and W. Pan, "Multiangle Social Network Recommendation Algorithms and Similarity Network Evaluation", Journal of Applied Mathematics, 2013 (2013).

[4] Y. Geng and K. Pahlavan, "On the accuracy of rf and image processing based hybrid localization for wireless capsule endoscopy", IEEE Wireless Communications and Networking Conference (WCNC), (2015).

[5] T. Su, W. Wang and Z. Lv, "Rapid Delaunay triangulation for randomly distributed point cloud data using adaptive Hilbert curve”, Computers \& Graphics, vol. 54, (2016), pp. 65-74.

[6] J. Hu, Z. Gao and W. Pan, "Multiangle Social Network Recommendation Algorithms and Similarity Network Evaluation", Journal of Applied Mathematics, 2013 (2013).

[7] S. Zhou, L. Mi, H. Chen and Y. Geng, "Building detection in Digital surface model", 2013 IEEE International Conference on Imaging Systems and Techniques (IST), (2012).

[8] K. Wang, X. Zhou and T. Li, "Optimizing load balancing and data-locality with data-aware scheduling", Big Data (Big Data), 2014 IEEE International Conference on. IEEE, (2014), pp. 119-128.

[9] L. Zhang, B. He and J. Sun, "Double Image Multi-Encryption Algorithm Based on Fractional Chaotic Time Series", Journal of Computational and Theoretical Nanoscience, vol. 12, (2015), pp. 1-7. 
[10] T. Su, Z. Lv and S. Gao, "3d seabed: 3d modeling and visualization platform for the seabed", Multimedia and Expo Workshops (ICMEW), 2014 IEEE International Conference on IEEE, (2014), pp. 1-6.

[11] Y. Geng, J. Chen, R. Fu, G. Bao and K. Pahlavan, "Enlighten wearable physiological monitoring systems: On-body rf characteristics based human motion classification using a support vector machine", IEEE transactions on mobile computing, vol. 1, no. 1, (2015), pp. 1-15.

[12] Z. Lv, A. Halawani and S. Feng, "Multimodal hand and foot gesture interaction for handheld devices", ACM Transactions on Multimedia Computing, Communications, and Applications (TOMM), vol. 11, no. 1 s, (2014), p. 10.

[13] G. Liu, Y. Geng and K. Pahlavan, "Effects of calibration RFID tags on performance of inertial navigation in indoor environment", 2015 International Conference on Computing, Networking and Communications (ICNC), (2015).

[14] W. Gu, Z. Lv, M. Hao, "Change detection method for remote sensing images based on an improved Markov random field", Multimedia Tools and Applications, (2015), pp. 1-16.

[15] Z. Chen, W. Huang and Z. Lv, "Towards a face recognition method based on uncorrelated discriminant sparse preserving projection”, Multimedia Tools and Applications, (2015), pp. 1-15.

\section{Authors}

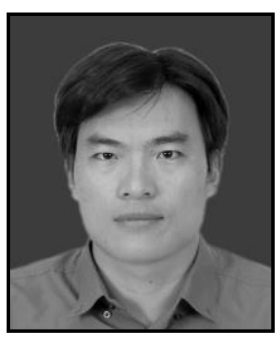

Li Mou Xu, he was born in ShanDong, China, in 1981. He received M.S. degree in computer application technology from Hunan University of Technology, Zhuzhou, China, in 2005 and 2008. $\mathrm{He}$ is a lecturer and senior engineer at Zhongshan Torch Polytechnic. His research interests include Computer application technology and Internet of things technology. 
International Journal of Future Generation Communication and Networking Vol. 9, No.8, (2016) 